\section{Breaking Onion Bulb Endodormancy with Hydrogen Peroxide}

\author{
Christopher J. D'Angelo \\ Illinois Foundation Seeds, Inc., Plover, WI
}

Irwin L. Goldman

Department of Horticulture, University of Wisconsin-Madison, 1575 Linden

Drive, Madison, WI 53706

Additional index words. Allium cepa, breeding, dormancy, vernalization

\begin{abstract}
Long-day storage onion bulbs (Allium cepa) undergo a period of endodormancy that begins before harvest and lasts for several weeks, depending on the genotype. Onion seed production relies on cold treatment to break endodormancy and vernalize the bulbs. When bulbs are planted shortly after harvest, endodormancy results in delayed growth and, in turn, slower flowering and seed production. Through this work we sought to explore the use of hydrogen peroxide as a treatment to break dormancy in onion bulbs. Endodormant bulbs of two long-day cultivars, Cortland and Sherman, were treated with hydrogen peroxide solutions at various concentrations in a series of experiments over a 3year period and were monitored for root and leaf (sprout) development. We found a 2- to 4-hour exogenous treatment of $20 \%$ (weight by volume) hydrogen peroxide to be highly effective at initiating uniform root growth in endodormant bulbs. When compared with a purified water control, the $20 \%$ treatment resulted in a $61.3 \%$ average reduction in the time to rooting in 2016. We also observed improved uniformity in rooting time between 'Cortland' and 'Sherman' in all 3 years of this work. We propose this novel method as a tool for breeders, researchers, and seed producers seeking rapid, uniform endodormancy release in onion bulbs to hasten seed production.
\end{abstract}

Onion (Allium cepa) is a globally important vegetable crop. It is grown in more than 160 countries and on more than 4.9 million ha. Global onion production has been increasing steadily since 1990 (FAO, 2016). The most widely produced marketable product of common onion is a dormant bulb. It is important for the bulbs to remain unsprouted while in storage because premature sprouting results in poor-quality bulbs with low consumer acceptance. Dormancy plays an integral role in onion bulb storage longevity and sprout suppression, but can also delay growth when it is desired. Dormancy release, along with vernalization, are key processes for seed production during which rapid, uniform growth and development are valuable. There is great economic interest in controlling dormancy release for both breeding and seed production.

Dormancy is a temporary suspension of visible growth of any plant structure containing a meristem (Lang et al., 1987). There are three classifications of dormancy, which are

Received for publication 9 Apr. 2019. Accepted for publication 29 July 2019.

We thank Ned, John, and Joan Crescio of Jack's Pride Farms in Randolph, WI, for their assistance and for donating the bulbs used in this study.

We claim no affiliation with Acros Organics, BDH, or their respective parent companies, Thermo Fisher Scientific and VWR International/Avantor. This study does not constitute or imply an endorsement of either company or their products.

I.L.G. is the corresponding author. E-mail: ilgoldma@wisc.edu. defined by the origin of initial growth suppression cues: endodormancy, paradormancy, and ecodormancy. Endodormancy occurs when the signal to suspend growth originates within the affected structure. Paradormancy is a response to a signal that originates in an organ other than the affected structure; ecodormancy is a response to unfavorable and extreme environmental conditions, such as very high or low temperatures or water availability (Lang et al., 1987). All three types of dormancy may occur simultaneously within a plant, but the type present in each structure depends on the life stage, physiology, and environmental conditions. For onion bulbs that have been harvested and put into cold storage, endodormancy and ecodormancy are the primary processes that regulate growth (Chope et al., 2012a).

Bulb endodormancy is prevalent in longday storage onion germplasm and begins to take effect in the weeks before harvest. As onions growing in the field complete their bulbing phase, they cease production of new leaves and the remaining leaves topple as they senesce. This senescence is often used by growers as an indication that the bulbs are ready to be harvested. When removed from the field, bulbs are taken into storage where they remain dormant. After $\approx 3$ weeks, the bulbs resume preharvest levels of cellular division and transcription (Chope et al., 2012b; Pak et al., 1995). Pak et al. (1995) found that as cellular division resumes, bulbs are capable of rooting when exposed to high moisture. This marks the transition from endodormancy to ecodormancy.
In onion, endodormancy is typically broken through exposure to cold temperatures. These cold treatments also serve to vernalize the bulbs. Little is known about the mechanism of endodormancy release in most plant species, but in seeds, which have been investigated most thoroughly, there is generally a strong association with abscisic acid degradation and gibberellic acid synthesis as dormancy is broken (Née et al., 2017). Successful use of chemical treatments to break endodormancy in seeds and the buds of woody plants using compounds such as hydrogen cyanamide have been reported (Horvath et al., 2003; Mohamed et al., 2012; Vergara and Pérez, 2010). Hydrogen cyanamide acts as a catalase inhibitor, which is a key enzyme that removes reactive oxygen species from plant cells. When administered to endodormant grapevine buds, hydrogen cyanamide was shown to increase levels of hydrogen peroxide in cells before breaking dormancy (Mazzitelli et al., 2007; Mohamed et al., 2012; Pérez and Lira, 2005). Pérez and Lira (2005) hypothesized that hydrogen peroxide acts as a secondary messenger to signal the release of endodormancy in grapevines (Vitis vinifera L.). This hypothesis was later supported by Mohamed et al. (2012) upon finding that, before dormancy release, an accumulation of hydrogen peroxide causes a temporary oxidative stress in grape buds following an exogenous application of hydrogen cyanamide. Similar hypotheses have been posited regarding hydrogen peroxide's role as a signal in seed dormancy release (Oracz et al., 2007). Chemical signaling under stress is a common role for reactive oxygen species, including hydrogen peroxide. Specifically, hydrogen peroxide has been shown to play a role in a range of cellular processes including programmed cell death, response to wounding, and abscisic acid-mediated stomatal closure (ElMaarouf-Bouteau and Bailly, 2008). An exogenous application of hydrogen peroxide has also been used successfully to break dormancy in seeds (Liu et al., 2011). Much like the findings in endodormant buds and seeds of other plant species, transcriptional analysis of onion bulbs during dormancy release shows that transcripts associated with genes related to defense and stress response are highly upregulated during the transition from endodormancy to ecodormancy (Chope et al., 2012b).

The hypothesis by Pérez and Lira (2005), in combination with the demonstrated success in using hydrogen peroxide to break seed dormancy, made it an interesting candidate for use on onion bulbs. Through this work, we sought to test the effects of an exogenous application of hydrogen peroxide on endodormant onion bulbs and to determine whether these treatments could serve as an effective way to break endodormancy.

\section{Materials and Methods}

Field design. A series of experiments to test the effects of exogenous hydrogen peroxide 
treatments on onion bulb endodormancy was conducted from 2014 to 2016. The 2014 experiment was conducted as a pilot study. Bulbs of the $F_{1}$ hybrid cultivars Cortland and Sherman (Bejo Seeds, Oceano, CA) were used in all years of this experiment. The bulbs were grown under commercial growing conditions on muck soil at Jack's Pride Farm in Randolph, WI, and were replicated at a second location in Markesan, WI, during 2015 and 2016. Seed was planted between late April and early May on raised beds at a density of 36 plants/ $\mathrm{m}$ using a modified Planet Junior (Cole Planter Company, Albany, GA) planter equipped with a cone seeder attachment in $3.66-\mathrm{m}$ rows with a $1.22-\mathrm{m}$ alley and $30-\mathrm{cm}$ row spacing. No maleic hydrazide or other sprout inhibitors were applied to the plants in the field. All bulbs were topped in the field, harvested in early September, and cured for 1 week in a dark, well-ventilated storage room at ambient temperature. Bulbs from the 2014 experiment were only harvested from the Randolph, WI, location. Six bulbs per treatment, cultivar, and location were used in all years of this study; however, in 2016, there were two replications per location from which bulbs were harvested. The 2014 and 2015 experiments used bulbs that were harvested from only one replication per location. The diameter of each bulb was measured transversely at the widest point using Vernier calipers.

Chemical source. Two concentrated sources of hydrogen peroxide were used in these experiments. The Acros Organics hydrogen peroxide $50 \%$ by weight solution in water (AC302865000; Acros Organics, Geel, Belgium) was first used in 2014. In 2015, the $\mathrm{BDH}^{1}$ hydrogen peroxide $30 \%$ stabilized ACS (BDH7690-1; VWR, Radnor, PA) solution was used. Both products use additives to stabilize the hydrogen peroxide and to slow degradation of the product during storage. However, stabilizer use can differ by manufacturer, and each product used different stabilizers. The Acros Organics product is stabilized with up to $200 \mathrm{ppm}$ phosphate from an undisclosed source, whereas the BDH product uses sodium stannate. Following the 2015 experiment, the experiment in 2016 was conducted using both products to assess whether these differently stabilized solutions influenced dormancy release. For both products and in all years of this study, the hydrogen peroxide stock solution was diluted to the appropriate final concentration using purified water (E-pure Water Purification model 7117; Thermo Fisher Scientific, Waltham, MA) immediately before treating the bulbs.

Bulb treatment. The top third of each bulb was cut so that the basal plate and two thirds of the bulb remained. Bulbs were placed into a plastic container with the cut side facing down while the appropriate hydrogen peroxide solution was added to the container. Initially, in $2014,10 \%, 20 \%$, and $30 \%$ hydrogen peroxide solutions were tested and compared with a control group that used only purified water $(0 \%$ hydrogen peroxide). After finding all treatment groups to be effective in 2014 , a lower concentration was included in the subsequent experiments. A 4\% hydrogen peroxide solution was chosen to be included in the 2015 and 2016 experiments because this is typical of the concentration found in the consumergrade product and is widely available in stores across the United States. Enough solution was added to each container so that the cut side of the bulb was submerged in $\approx 1.5 \mathrm{~cm}$ of liquid (Fig. 1A). The bulbs were left to soak uncovered in the solution for $4 \mathrm{~h}$ at ambient temperature. An alternate 2-h soak was also evaluated in 2015 alongside the 4-h treatment. Upon completion of the treatment, the bulbs were removed from the solution and rinsed under cool tap water to remove any excess solution. The bulbs were then planted in a greenhouse in a completely randomized design (Fig. 1B). Bulbs were inspected every 2 to $3 \mathrm{~d}$ for rooting and sprouting. The date was recorded for the initial root and sprout observations for each plant.

Greenhouse culture. All bulbs were planted in black plastic pots. The potting media was a 2:1 mix of silty loam compost soil collected from the West Madison Agricultural Research Station and soilless medium (MetroMix 366; Sun Gro Horticulture, Agawam, MA). The greenhouse temperature in 2014 was kept at $16{ }^{\circ} \mathrm{C}$ using steam baseboard radiators and a combination of
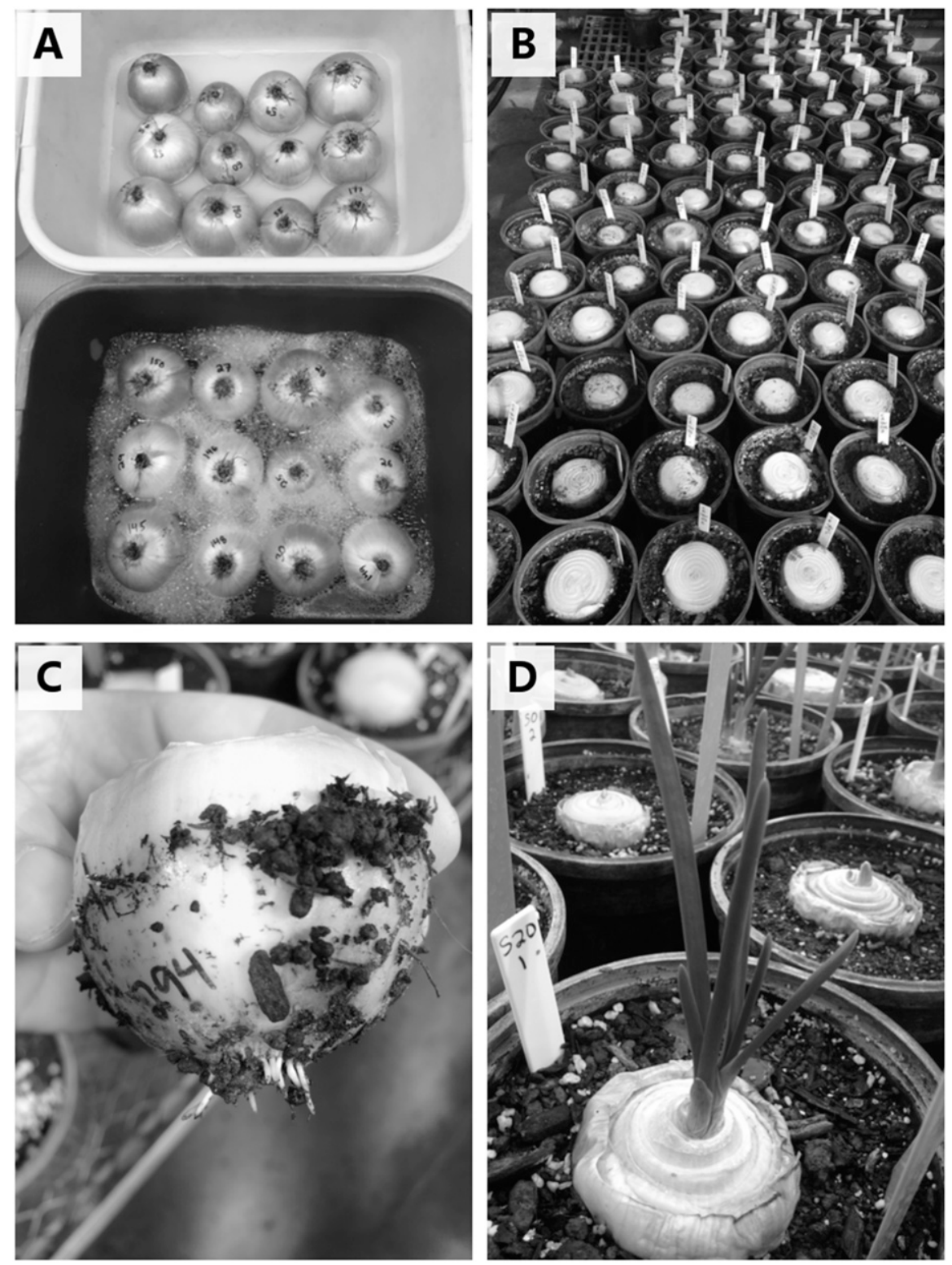

Fig. 1. (A) Cut onion bulbs soaking in 30\% hydrogen peroxide solutions from the 2016 experiment. The top bucket is filled with sodium stannate-stabilized hydrogen peroxide; the bottom bucket contains phosphate-stabilized hydrogen peroxide. Note the difference in foaming between the two solutions. (B) Onions planted in the greenhouse following treatment with hydrogen peroxide. (C) Rooting onion in the greenhouse. Bulbs were lifted from the soil to observe and record initial root growth. (D) Sprouting 'Sherman' onion from the $20 \%$ treatment group. 
manual and automated vents. In 2015-16, air-conditioned greenhouses with forced-air heating were used to maintain a temperature of $20^{\circ} \mathrm{C}$. In all years, high-pressure sodium supplemental lighting was used to maintain a 16-h daylength with a photon flux density of $103 \mu \mathrm{mol} \cdot \mathrm{m}^{-2} \cdot \mathrm{s}^{-1}$ in 2014 , and 343 $\mu \mathrm{mol} \cdot \mathrm{m}^{-2} \cdot \mathrm{s}^{-1}$ in 2015 and 2016 (Model QMSS; Apogee Instruments, Logan, UT). Plants were watered as needed daily and were fertilized as needed with a $400-\mathrm{mg} \cdot \mathrm{L}^{-1}$ mixture of $20 \mathrm{~N}-4.365 \mathrm{P}-16.602 \mathrm{~K}$ fertilizer with micronutrients (Peters Professional Peat-Lite Special; ICL Specialty Fertilizers, Dublin, $\mathrm{OH}$ ).

Data collection. Following treatment and subsequent planting in the greenhouse, bulbs were monitored every 2 to $3 \mathrm{~d}$ for signs of root development and sprouting (Fig. 1C and D). The date that each event was first observed was recorded.

Statistics. Natural logarithmic transformations were performed on all days-torooting and days-to-sprouting data. Individual bulbs were treated as experimental units with replication over time. Measuring individual bulbs allowed us to assess the response of bulb-to-bulb variation treatment with hydrogen peroxide. Analysis of variance (ANOVA) was performed using the 'car' package in $\mathrm{R}$ with type three sums of squares and effect sizes were measured using an $\eta^{2}$ calculation from the 'sjstats' package. Tukey's highly significant difference (HSD) test was also performed in R using the 'stats' package (R Development Core Team, 2018). Nonsignificant interaction terms, such as concentration:location and concentration:variety were not included in the linear models for this analysis.

\section{Results}

The 2014 and 2016 experiments found that 'Cortland' and 'Sherman' bulbs treated with $10 \%, 20 \%$, and $30 \%$ solutions of hydrogen peroxide exhibited expedited rooting and sprouting when compared with the $0 \%$ control group (Figs. 2-7). For 2016, the 20\% treatment resulted in a $61.3 \%$ average reduction in the time to rooting. These data show that the $20 \%$ hydrogen peroxide treatment was associated with the lowest mean time to rooting and sprouting, with little spread (Figs. 2, 5, and 6). Except for 'Cortland' bulbs harvested from Markesan in 2015, all varieties and treatment durations showed fast, uniform rooting after the $20 \%$ treatment. The sprouting data show a similar trend, although the spread of the data for each treatment group is larger than for root development. There was no apparent difference in time from rooting to sprouting across treatment groups in 2015 and 2016, although a weak negative trend was observed in 2014 as the treatment concentration increased (Fig. 7).

Although data from 2015 showed improvements in the time to rooting for some treatment groups, the trends are less clearly defined than in 2014 and 2016 (Figs. 3 and

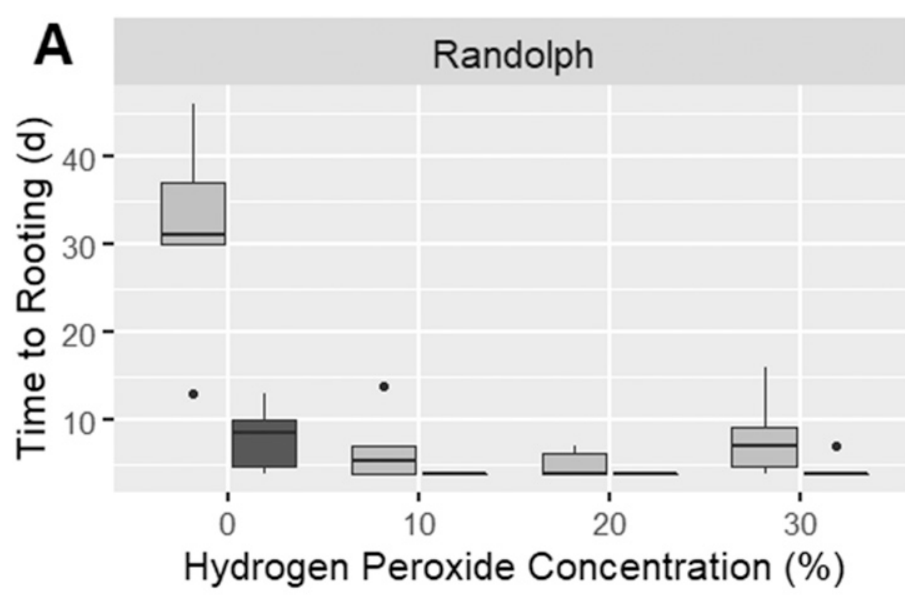

Cultivar

Cortland

Sherman

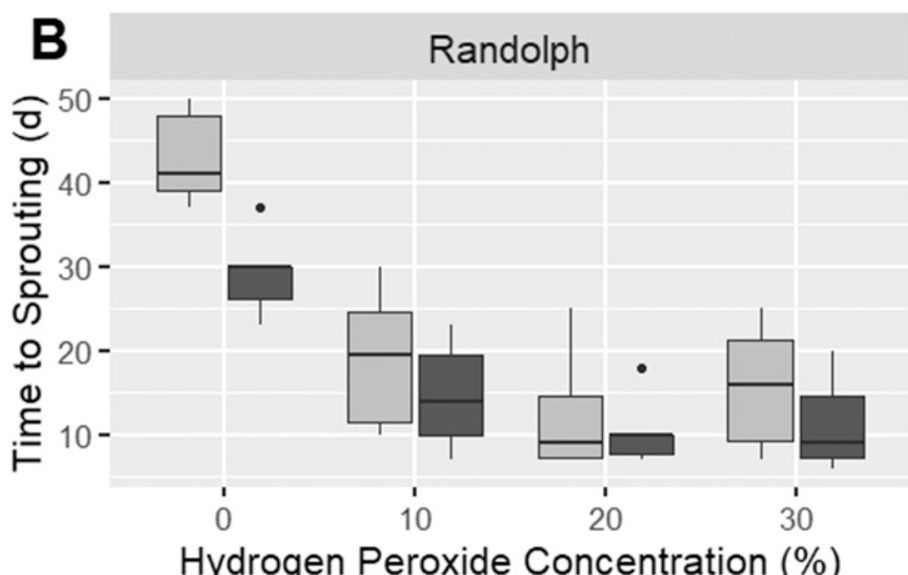

Cultivar

Cortland

Sherman

Fig. 2. Effects of hydrogen peroxide on onion bulb dormancy from the initial 2014 experiment. (A) Comparison of the time to rooting (days) after treatment with hydrogen peroxide in the $F_{1}$ hybrid cultivars Cortland and Sherman. (B) Comparison of the time to sprouting (days) after being treated with hydrogen peroxide in the $\mathrm{F}_{1}$ hybrid cultivars Cortland and Sherman. Dots on the boxplots indicate values of potential outliers that exceeded 1.5 times the interquartile range.

Table 1. Degrees of freedom (df) and F values from an analysis of variance (ANOVA) for time to rooting (measured in days) in 'Cortland' and 'Sherman' after treatment with hydrogen peroxide solutions at five concentrations in the 2015 and 2016 experiments.

\begin{tabular}{|c|c|c|c|c|c|c|}
\hline \multirow{2}{*}{$\begin{array}{l}\text { ANOVA } \\
\text { Source }\end{array}$} & \multicolumn{3}{|c|}{2015} & \multicolumn{3}{|c|}{2016} \\
\hline & $\mathrm{df}$ & & & $\mathrm{df}$ & & \\
\hline Concentration & 4 & 5.35 & $* * *$ & 4 & 36.05 & k** \\
\hline Cultivar & 1 & 26.96 & $* * *$ & 1 & 32.78 & *** \\
\hline Location & 1 & 4.69 & $*$ & 1 & 12.22 & *** \\
\hline Bulb diameter & 1 & 2.44 & & 1 & 30.16 & *** \\
\hline Stabilizer & - & - & & 1 & 4.16 & * \\
\hline Replication & - & - & & 1 & 0.04 & \\
\hline Hours & 1 & 2.63 & & - & - & \\
\hline Cultivar:location & 1 & 0.02 & & 1 & 10.76 & ** \\
\hline Residuals & 171 & & & 453 & & \\
\hline
\end{tabular}

$*, * *, * * *$ Significant at $P \leq 0.05,0.01$, or 0.001 , respectively.

$-=$ Terms not included in the linear model for the given analysis.

4). These data showed greater variances at the $10 \%, 20 \%$, and $30 \%$ concentration treatment groups for time to rooting than in 2014 and 2016 (Fig. 3). In addition, there is a reduced effect on the mean time to sprouting across all treatment groups when compared with the $0 \%$ control (Fig. 4). In both 'Cortland' and 'Sherman', a $4 \%$ treatment, regardless of duration, increased the time to sprouting compared with the control. Similarly, the $30 \%$ treatment in 2015 resulted in very poor uniformity in the time to rooting, particularly in bulbs harvested from Randolph.

'Cortland' and 'Sherman' bulbs averaged rooting in $11.1 \mathrm{~d}$ and sprouting in $28.6 \mathrm{~d}$ across two environments after a 4 -h treatment with $20 \%$ hydrogen peroxide in 2016 . There is a strong varietal main effect in the time to rooting and, to a lesser degree, in the time to sprouting (Table 1). The greatest difference between the two varieties can be observed in the control and $4 \%$ treatment groups. 


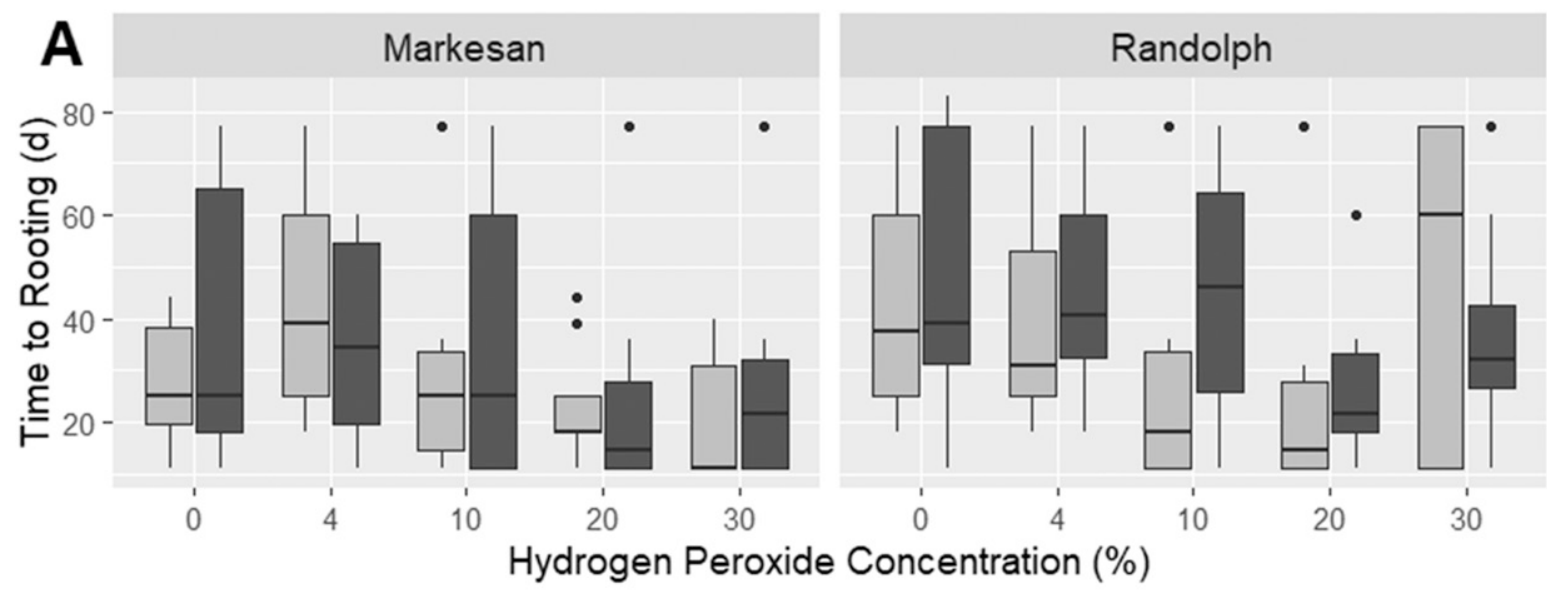

Treatment Duration

2 Hours

4 Hours

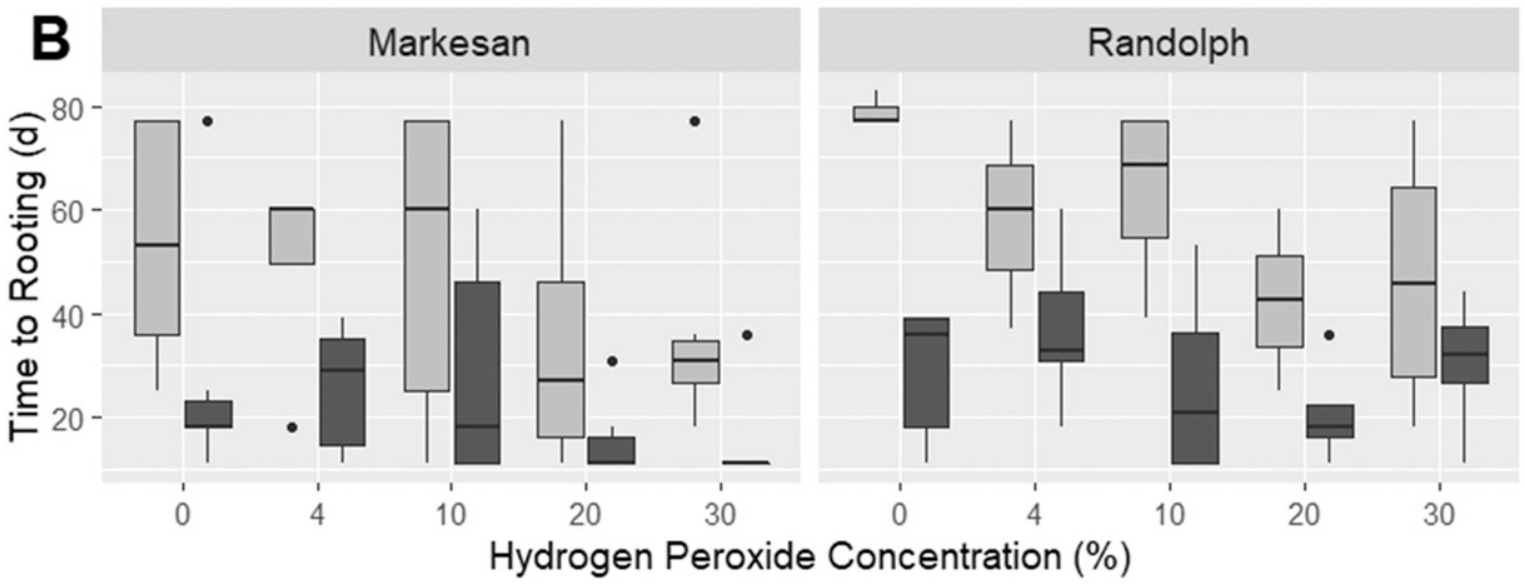

\section{Cultivar

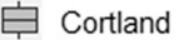 \\ Sherman}
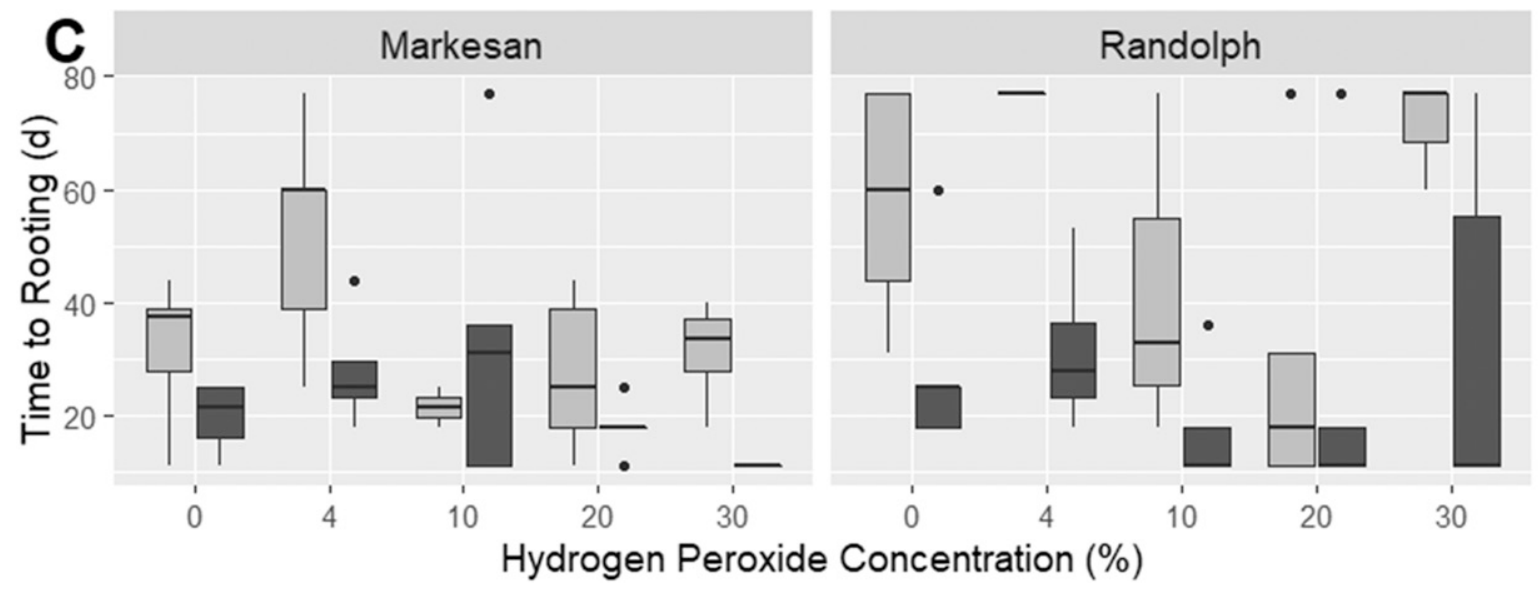

Cultivar

Cortland

Sherman

Fig. 3. Time to rooting (days) in bulbs harvested from Markesan and Randolph, WI, in 2015. (A) Comparison of the time to rooting (days) with 2-h and 4-h treatment durations. Individual cultivar responses of $\mathrm{F}_{1}$ hybrids Cortland and Sherman to rooting time (days) following a (B) $4-\mathrm{h}$ and a $(\mathbf{C}) 2-\mathrm{h}$ treatment with hydrogen peroxide. Dots on the boxplots indicate values of potential outliers that exceeded 1.5 times the interquartile range.

'Cortland' had a much stronger response to hydrogen peroxide than 'Sherman', although after the $10 \%$ and $20 \%$ treatments in 2014 and 2016 there was little difference between the two varieties. 'Cortland' and 'Sherman' differed by just $2.1 \mathrm{~d}$ for rooting and $4.9 \mathrm{~d}$ for sprouting in the $20 \%$ treatment, whereas the control differed by $21.0 \mathrm{~d}$ for rooting and $17.6 \mathrm{~d}$ for sprouting.

Two-hour and 4-h treatment durations were tested in 2015. Although the data from the 4-h treatments in 2015 differed from other years, the 2-h treatment was effective and followed the trends observed in 2014 and 2016. There was considerable disease pressure in 2015 that resulted from an environmental effect between the two harvest locations in both treatment durations, but the magnitude of the effect was greater in the 2-h treatment. This effect is most clear in the $10 \%$ and $30 \%$ treatment groups, in which performance is vastly different in each environment at each treatment level (Fig. 6). Despite the environmental effects, Tukey's HSD test found a $20 \%$ hydrogen peroxide treatment to reduce rooting time significantly in both the 2015 and 2016 experiments when compared with a purified water control (Table 2).

It was noted that solutions using the phosphate-stabilized stock from BDH foamed considerably more than the sodium stannatestabilized stock after the bulbs were submerged (Fig. 1A). However, although the stabilizer term was significant $(\alpha \leq 0.05)$ in the ANOVA, the stabilizer-by-concentration interaction was not (Table 1). The effect size of the stabilizer term was also small, with an $\eta^{2}$ value of $0.52 \%$. 


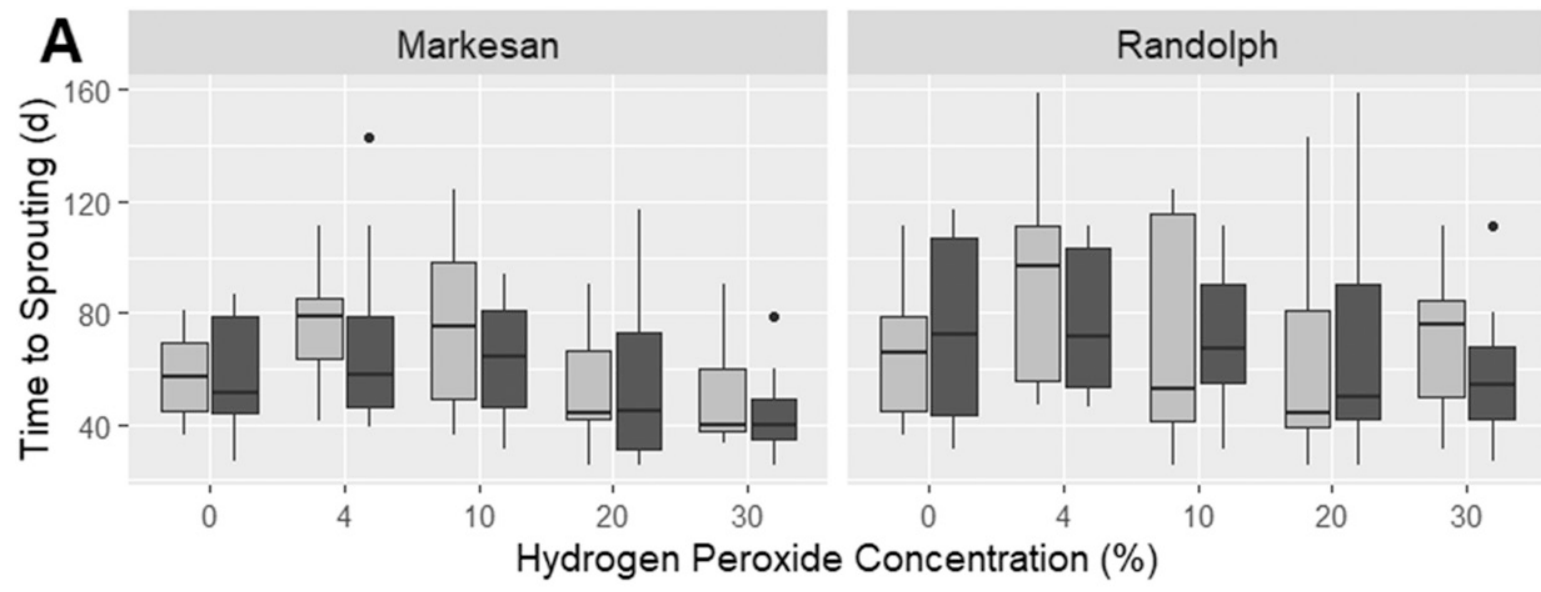

Treatment Duration

2 Hours

4 Hours
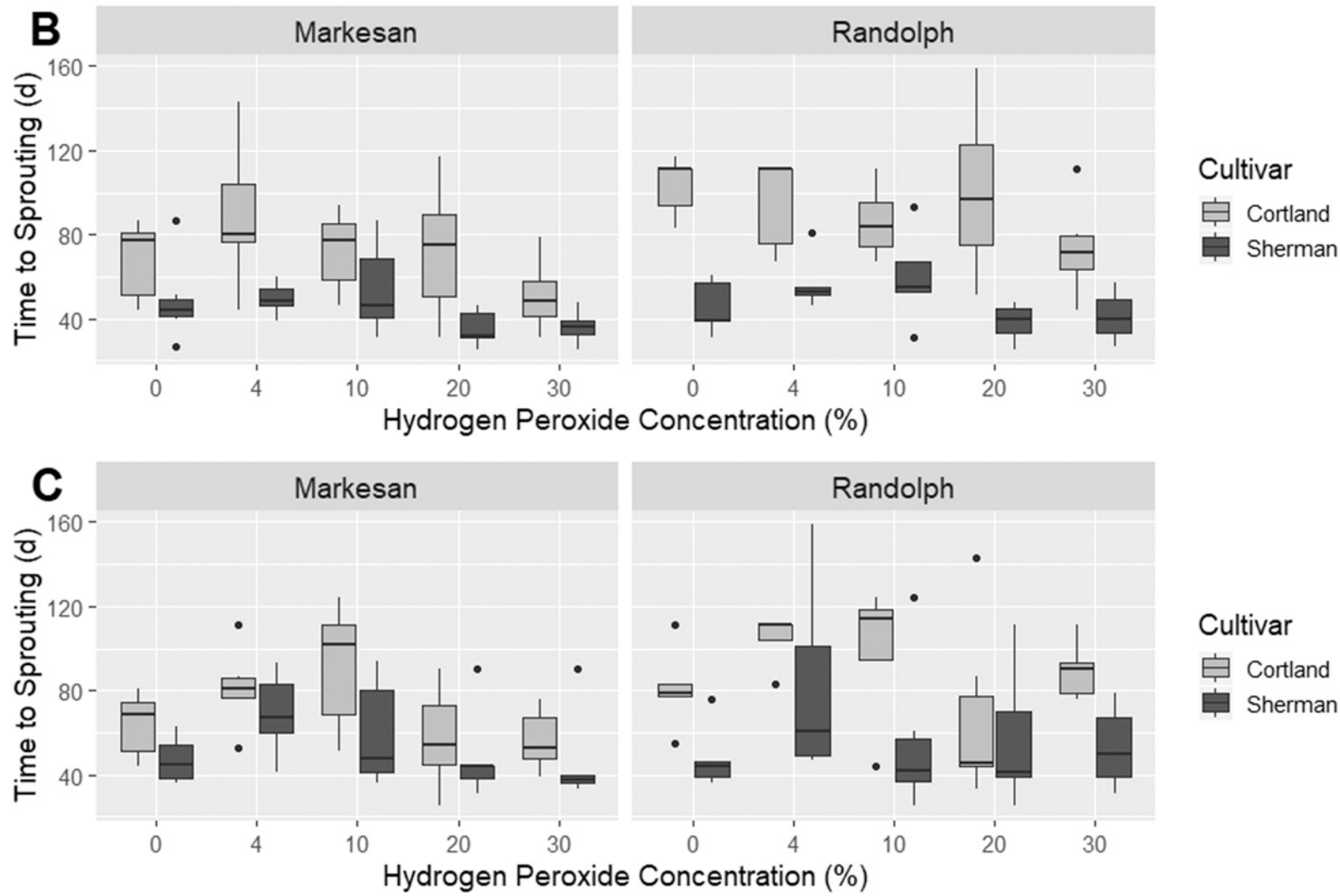

Cultivar

Cortland

Sherman

Fig. 4. Time to sprouting (days) in bulbs harvested from Markesan and Randolph, WI, in 2015. (A) Comparison of the time to sprouting (days) with 2-h and 4-h treatment durations. Individual cultivar responses of $\mathrm{F}_{1}$ hybrids Cortland and Sherman to sprouting time (days) following a (B) 4-h and a (C) 2-h treatment with hydrogen peroxide. Dots on the boxplots indicate values of potential outliers that exceeded 1.5 times the interquartile range.

Bulb diameter exhibited a significant effect on time to rooting in 2016, but not in 2015. The $\eta^{2}$ value indicates that bulb diameter only accounted for $3.4 \%$ of the variance in the time to rooting, which is likely a result, in part, of the uniformity of the bulbs in the experiment. The mean bulb diameter was $68.1 \pm 7.5 \mathrm{~mm}$ in 2016 and $72.6 \pm 8.1 \mathrm{~mm}$ in 2015 . The 2016 data revealed that each additional millimeter in bulb diameter resulted in a $2.8 \%$ average decrease in time to rooting.

Several of the bulbs in all treatment groups as well as the control sprouted before developing roots. This was not observed in
2014 , but was found in $3.9 \%$ of the bulbs that sprouted in 2015 and in $1.1 \%$ of the bulbs that sprouted in 2016. In some cases, root initiation began within several days of sprouting, but others produced roots after several weeks, if at all. There was no pattern to suggest that treatment concentration increases the likelihood of this phenomenon, but there was a clear varietal and stabilizer effect. A total of $78.6 \%$ of the bulbs in this group were from the cultivar Sherman and $93.3 \%$ were treated with sodium stannate-stabilized hydrogen peroxide.

Many of the plants grown in this study flowered without vernalization, which was an unexpected finding. A total of $86.1 \%$ of all 'Cortland' and $70.2 \%$ of all 'Sherman' bulbs grown in this study that sprouted also produced scapes, without being grown at vernalizing temperatures. This occurred in all 3 experimental years at high percentages. Excluding the data from 2014, which used slightly cooler greenhouse temperatures, the incidence of scape emergence increases to $88.2 \%$ in all 'Cortland' and $70.8 \%$ in all 'Sherman' bulbs. In 2016, on average, scapes emerged from 'Cortland' bulbs in $121.0 \pm 19.4 \mathrm{~d}$ and from 'Sherman' bulbs in $128.9 \pm 18.0 \mathrm{~d}$. 


\section{Discussion}

The presence of endodormancy in onion bulbs delays visible growth until dormancy is broken. Endodormancy is usually broken in onion bulbs after chilling for several weeks, but in the absence of chilling, dormancy break and initiation of visible growth is less uniform. As observed in this study, there are both varietal and environmental effects that affect the time to rooting and sprouting (Figs. 5-7). For growth in controlled envi- ronments where uniformity is desirable, differences in growth from year to year and across genotypes presents a challenge. A primary goal of this study was to determine whether an exogenous application of hydrogen peroxide to endodormant onion bulbs

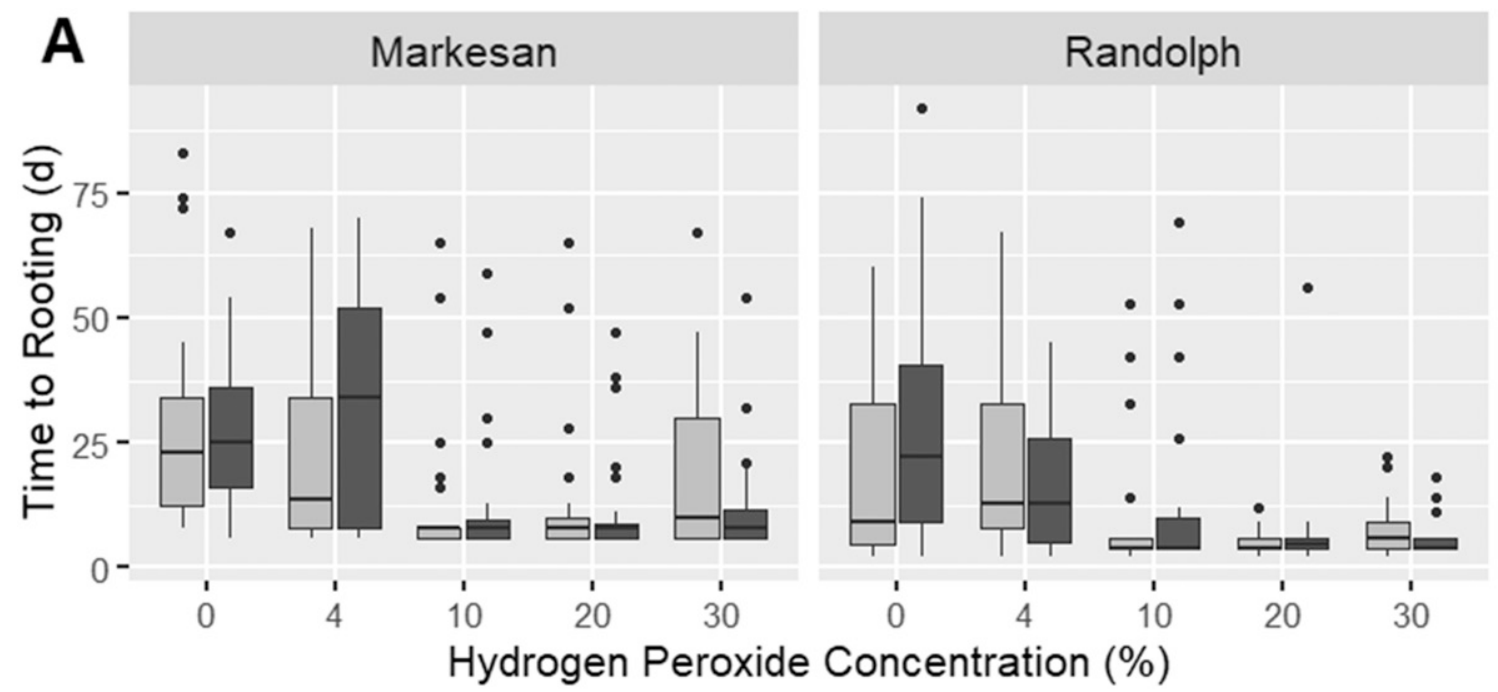

Stabilizer

Phosphate

Sodium

Stannate
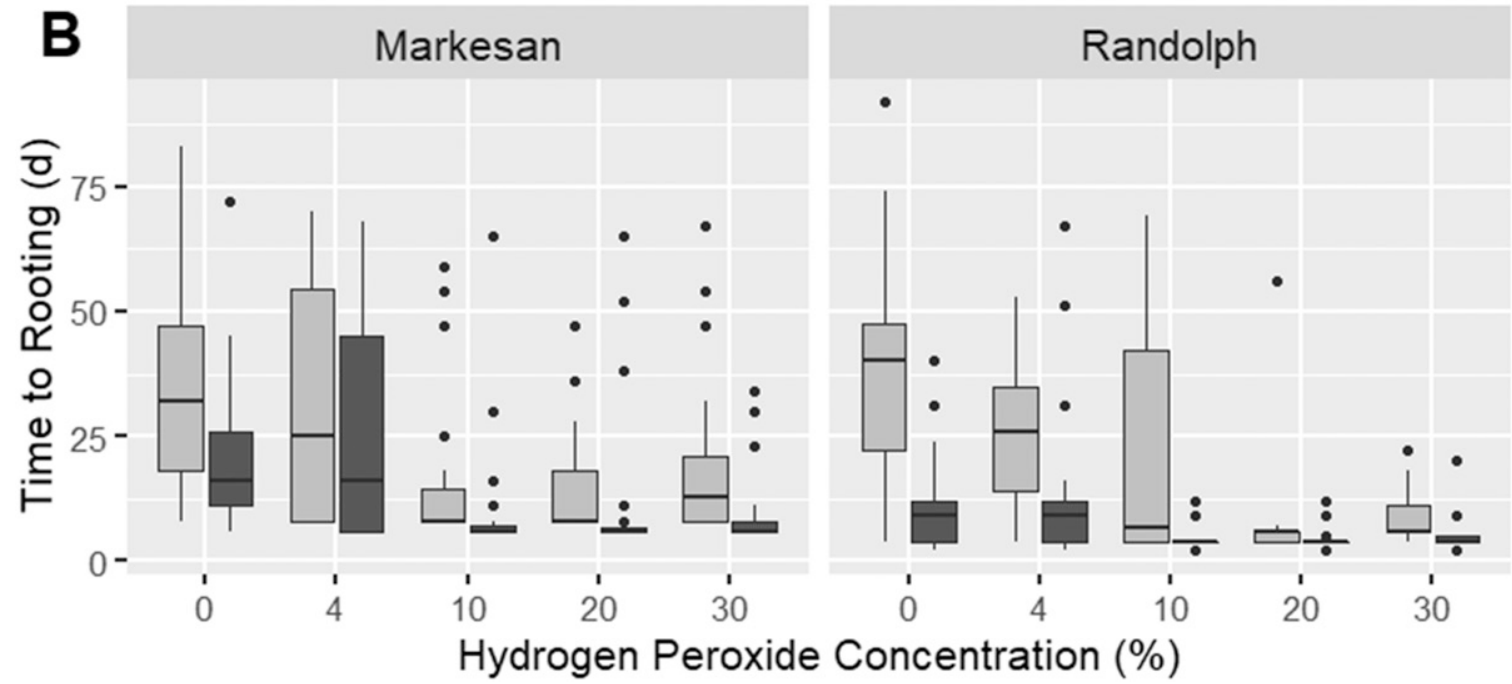

Cultivar

Cortland

Sherman

Fig. 5. Time to rooting (days) in bulbs harvested from Markesan and Randolph, WI, in 2016. (A) Comparison of the time to rooting (days) with phosphate and sodium stannate-stabilized hydrogen peroxide stock solutions across both cultivars. (B) Individual cultivar responses of $\mathrm{F}_{1}$ hybrids Cortland and Sherman to rooting time following a 4-h treatment with hydrogen peroxide. Note the difference in rooting time response across the two locations at lower treatment concentrations. Dots on the boxplots indicate values of potential outliers that exceeded 1.5 times the interquartile range.

Table 2. Tukey's honestly significant difference test results for time to rooting in the 2015 and 2016 experiments. Each treatment concentration is compared to determine whether the difference in rooting time is significantly different from zero. Natural logarithmic transformations of the rooting time data were used in these tests.

\begin{tabular}{|c|c|c|c|c|c|c|}
\hline \multirow{2}{*}{$\begin{array}{l}\text { Comparison of } \\
\text { concn treatments }\end{array}$} & \multicolumn{3}{|c|}{2015} & \multicolumn{3}{|c|}{2016} \\
\hline & Difference & $P$ value & Significance & Difference & $P$ value & Significance \\
\hline $4 \%$ to $0 \%$ & 0.0838 & 0.9740 & & -0.1655 & 0.5652 & \\
\hline $10 \%$ to $0 \%$ & -0.1811 & 0.6729 & & -0.8260 & 0.0000 & $* * *$ \\
\hline $20 \%$ to $0 \%$ & -0.4712 & 0.0054 & $* *$ & -1.0165 & 0.0000 & $* * *$ \\
\hline $30 \%$ to $0 \%$ & -0.2860 & 0.1896 & & -0.8208 & 0.0000 & $* * *$ \\
\hline $10 \%$ to $4 \%$ & -0.2649 & 0.3475 & & -0.6605 & 0.0000 & $* * *$ \\
\hline $20 \%$ to $4 \%$ & -0.5550 & 0.0012 & $* *$ & -0.8509 & 0.0000 & $* * *$ \\
\hline $30 \%$ to $4 \%$ & -0.3698 & 0.0611 & & -0.6553 & 0.0000 & $* * *$ \\
\hline $20 \%$ to $10 \%$ & -0.2901 & 0.2302 & & -0.1904 & 0.4303 & \\
\hline $30 \%$ to $10 \%$ & -0.1049 & 0.9374 & & 0.0053 & 1.0000 & \\
\hline $30 \%$ to $20 \%$ & 0.1852 & 0.6357 & & 0.1957 & 0.4253 & \\
\hline
\end{tabular}

**, ***Significant at $P \leq 0.01$ or 0.001 , respectively. 


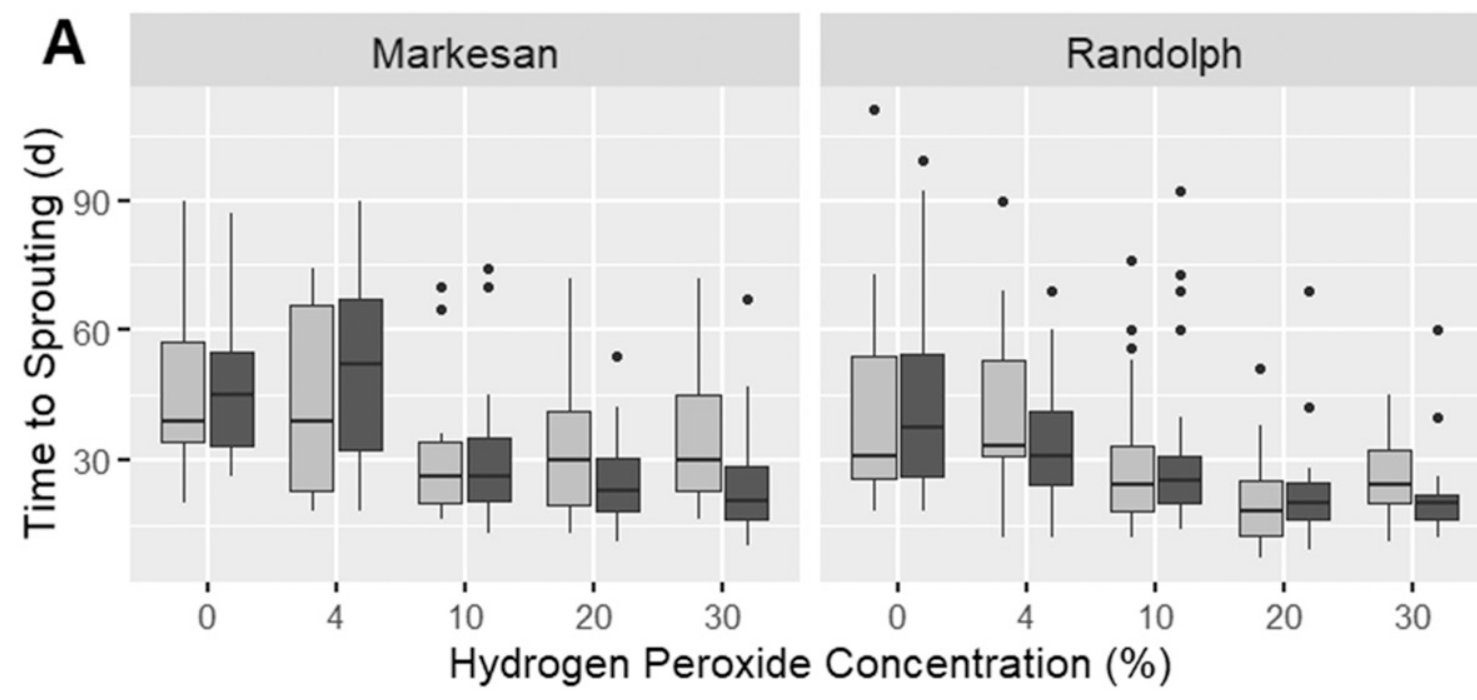

Stabilizer

Phosphate

Sodium

Stannate
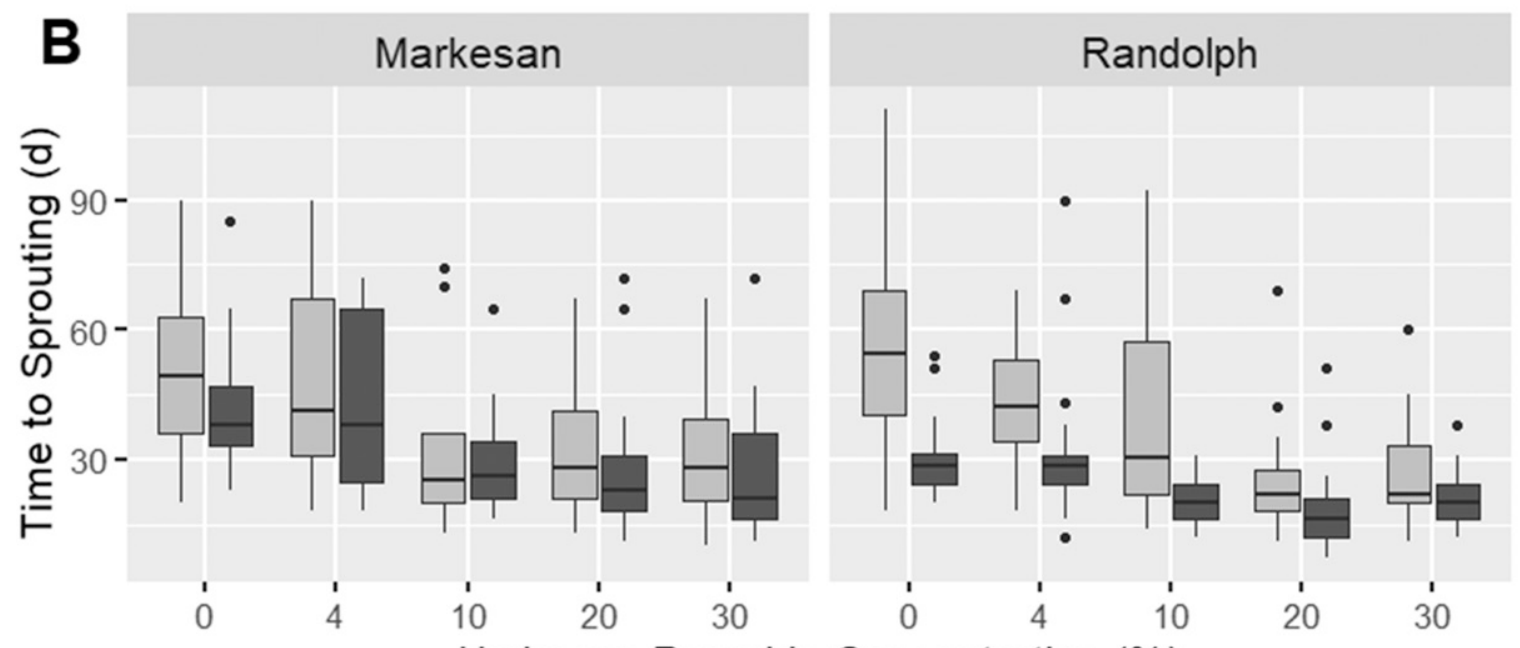

Cultivar

Cortland

Sherman

\section{Hydrogen Peroxide Concentration (\%)}

Fig. 6. Time to sprouting (days) in bulbs harvested from Markesan and Randolph, WI, in 2016. (A) Comparison of the time to sprouting (days) with phosphate and sodium stannate-stabilized hydrogen peroxide stock solutions across both cultivars. (B) Individual cultivar responses of $\mathrm{F}_{1}$ hybrids Cortland and Sherman to sprouting time following a 4-h treatment with hydrogen peroxide. Dots on the boxplots indicate values of potential outliers that exceeded 1.5 times the interquartile range.

would break dormancy and elicit uniform growth when planted in a greenhouse.

Throughout the 3 years during which this study was conducted, we found soaking dormant bulbs in a $20 \%$ hydrogen peroxide solution for 2 to $4 \mathrm{~h}$ to be an effective method for breaking dormancy (Table 2). Treating with a $20 \%$ solution reduced the time to rooting and improved the uniformity of initial root development, which signified that dormancy was broken (Lang et al., 1987). We also found that the time to sprouting is decreased with a $20 \%$ hydrogen peroxide treatment; however, this finding was attributed primarily to the treatment's effect on root development because there was no apparent difference in the time from rooting to sprouting between the control and any of the treatment groups in 2015 and 2016 (Fig. 7). By breaking dormancy and initiating growth earlier, these plants appear to be sprouting faster, but are progressing from rooting to sprouting at the same rate as the control.
The results from 2014 and 2016 demonstrate the effectiveness of the $20 \%$ treatment on minimizing varietal differences in the time to root development (Figs. 2 and 5). The difference in mean time to rooting between 'Cortland' and 'Sherman' in 2016 was $21.0 \mathrm{~d}$ in the control, but was reduced to $2.1 \mathrm{~d}$ in the $20 \%$ treatment group. 'Sherman' is generally considered to be earlier maturing than 'Cortland', so the difference in rooting time between the two varieties in the control group was consistent with our expectations. However, these results suggest that this treatment may break dormancy synchronously across a range of long-day genotypes.

The 2015 results were a departure from the very clear effects of the $10 \%$ to $30 \%$ treatment groups observed in 2014 and 2016. Although many of the trends that were identified in the other years are still present in the 2015 data, they were less clearly defined in the 4-h treatment and were muted in the 2-h treatment. The experimental design and methods were the same between 2014 and 2015, only expanded to include a second location and two treatment durations. However, there were two factors that could have contributed to these differences in results. The first was that a different brand of hydrogen peroxide was used in 2015 than in 2014. At the time, we were unaware that hydrogen peroxide solutions were stabilized and that the stabilizing compound differed by manufacturer. After discovering that each stock solution used a different stabilizer, both products were included in the 2016 experiment. Despite the significance of the stabilizer term in the ANOVA (Table 1), a paired $t$ test found that the difference between stabilizers for rooting time in the $10 \%, 20 \%$, and $30 \%$ treatment groups was not significant at the $\alpha=0.05$ level. This suggests that the use of differentially stabilized hydrogen peroxide solutions between 2014 and 2015 was likely not a major contributor to the difference in results. The second factor worth considering is that there was high disease pressure at both Randolph and Markesan in 2015. Both 

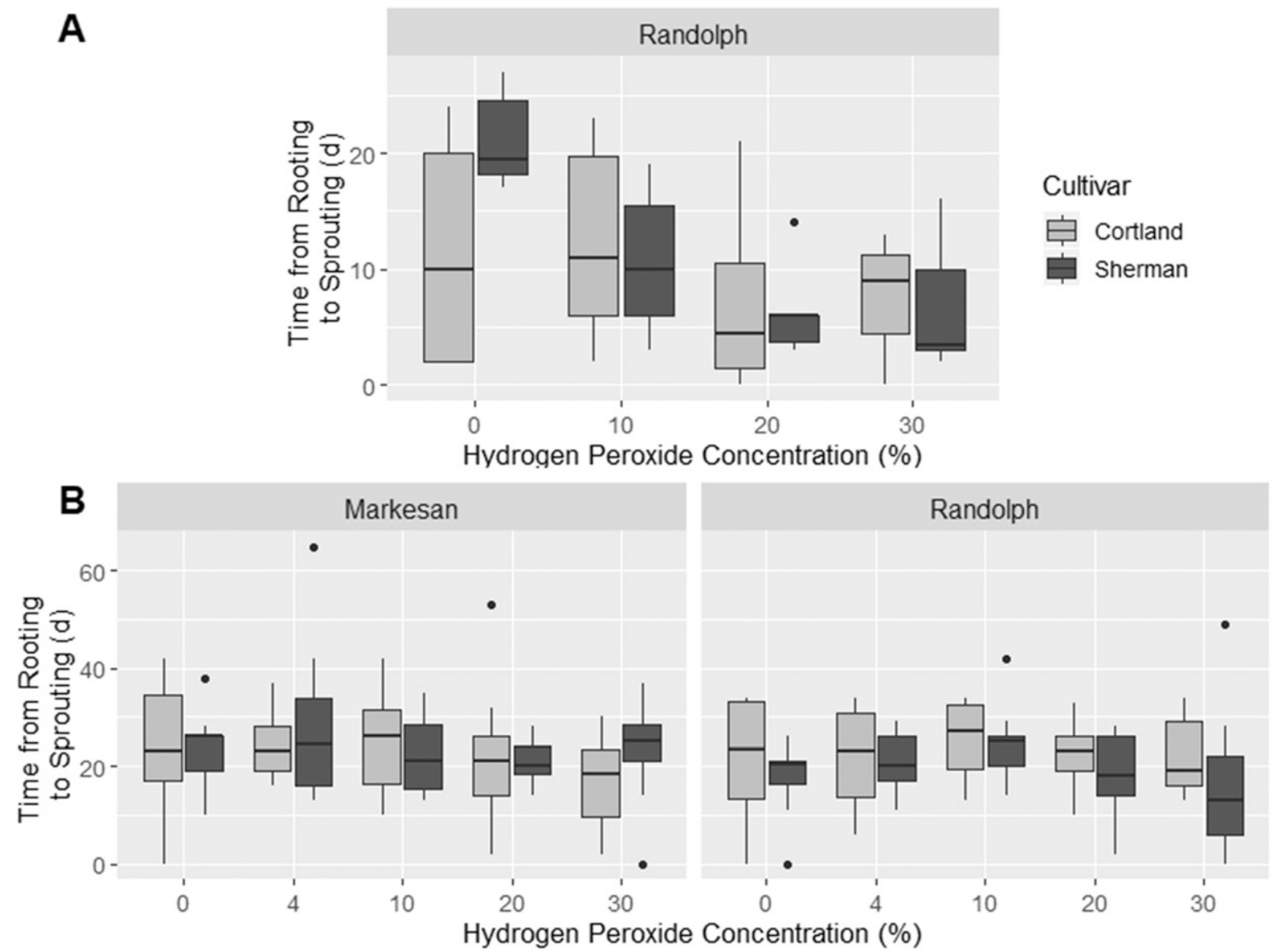

Cultivar
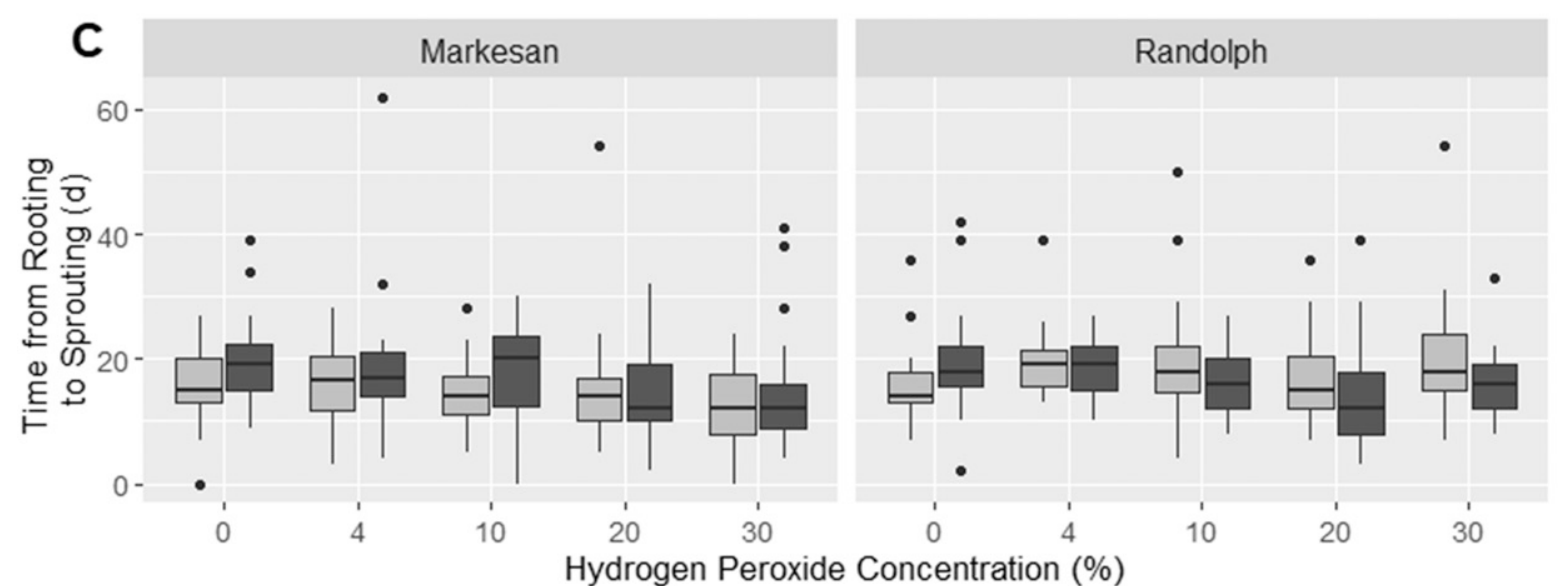

Cultivar

Sherman

Fig. 7. Boxplot of the difference in time from rooting to sprouting (days) for all 4-h treatments from the (A) 2014, (B) 2015 , and (C) 2016 experiments. Note that in 2015-16 there were no apparent differences in this transition time across different treatment concentrations. Dots on the boxplots indicate values of potentia outliers that exceeded 1.5 times the interquartile range.

locations had a high incidence of Stemphylium leaf blight (Stemphylium vesicarium), and many of our plots showed symptoms of the disease. Consequently, the bulbs used in the 2015 experiment were harvested $\approx 1$ week earlier than normal. As a result of the nature of the disease, most of the plants had leaves that were still standing upright despite being highly senesced. It is likely that this extensive disease pressure is the primary source of the differences in these results. Interestingly, the 2-h treatment was more effective at reducing the time to rooting than the 4-h treatment in 2015, particularly at the
$10 \%$ and $20 \%$ treatment levels, although only the $20 \%$ treatment was found to decrease rooting time significantly when compared with the control group (Table 2).

Another interesting observation was that the majority of plants, of both varieties, grown in these experiments produced scapes and flowered without being exposed to vernalizing temperatures. Vernalizing temperatures for onion range from 2 to $17{ }^{\circ} \mathrm{C}$ (Brewster, 2008). In 2014, the greenhouse temperature was kept at $16{ }^{\circ} \mathrm{C}$, which is within the upper end of the range of vernalizing temperatures, but still outside of the optimum range of 7 to $12{ }^{\circ} \mathrm{C}$, as reported by Brewster (2008). We concluded that the plants in 2014 were likely being vernalized as they grew in the greenhouse. However, the 2015 and 2016 experiments were conducted in a warmer $20{ }^{\circ} \mathrm{C}$ greenhouse with better temperature control than the greenhouse from 2014. The newer greenhouse used forced-air heating and air-conditioning combined with automated ridge vents to regulate temperature. Despite the new conditions and growing environment, $88.2 \%$ of all 'Cortland' and $70.8 \%$ of all 'Sherman' bulbs from 2015 and 2016 flowered. This finding is 
deserving of further investigation. If vernalization in long-day onion is facultative, rather than obligate, procedures might be designed to reduce the time necessary to complete the onion plant's life cycle (D'Angelo and Goldman, 2018). This, in turn, would hasten seed production and many breeding methods.

The $30 \%$ treatment effectively reduced the rooting time to levels that were comparable to the $10 \%$ and $20 \%$ treatments in 2014 and 2016. However, across all years, $\approx 20 \%$ of the bulbs in the $30 \%$ treatment became soft and rotten. The rot often also included mold that grew on the cut surface of the bulb and usually resulted in plant death or stunted growth. The $10 \%$ treatments were effective at reducing the rooting time in 2014, 2016, and in the 2015 2-h treatment group, but were sensitive to environmental effects and possibly genotype-by-environment interactions (Figs. 3C and 5B). In the 2-h 10\% treatment group from 2015, there was a change in rank for rooting time between 'Cortland' and 'Sherman' over the two harvest locations: Markesan and Randolph. The 20\% treatment consistently had the best performance across years, harvest locations, treatment durations, and in each of the varieties. In addition, the $20 \%$ treatment had the effect of breaking dormancy in 'Cortland' and 'Sherman' within $2.1 \mathrm{~d}$ of each other, on average. We have found the $20 \%$ treatment to be an effective, versatile tool for breaking dormancy in onion bulbs.

The ability to break dormancy in bulbs uniformly is an important advance for onion research. The novel method we are proposing offers researchers greater control over the timing and duration of treatments to onions grown from bulbs and may aid in offering insight to the molecular control of dormancy release. In addition, it has potential for synchronizing growth in diverse germplasm, which can be beneficial in the context of research, breeding, and seed production. There is an economic interest in synchronous growth in the seed industry because asynchronous growth affects seed yield negatively and limits cross-pollinations to plants at a similar reproductive maturity. This method may also prove to be a useful tool in the development of an annual-cycle breeding system that does not rely on seedling vernalization. Depending on the relationship between vernalization and dormancy, if nondormant bulbs can vernalize at a faster rate than dormant bulbs, as reported by Bertaud (1988) and Shishido and Saito (1977), then rapid, uniform dormancy release may be key in solving the annualization puzzle. Further research into the effects of treatment duration and the impact of high disease pressure on the efficacy of these treatments is necessary.

\section{Literature Cited}

Bertaud, D.S. 1988. Effects of chilling duration, photoperiod and temperature on floral initiation and development in sprouted and unsprouted onion bulbs, p. 254-261. In: Symposium on Allium: EUCARPIA - Section Vegetables, 4th Coventry 6-9 Sept. 1988 United Kingdom GB.

Brewster, J.L. 2008. Onions and other vegetable alliums. 2nd ed. Crop production science in horticulture 15. CAB International, Wallingford, UK.

Chope, G.A., K. Cools, J.P. Hammond, A.J. Thompson, and L.A. Terry. 2012a. Physiological, biochemical and transcriptional analysis of onion bulbs during storage. Ann. Bot. 109:819-831.

Chope, G.A., K. Cools, L.A. Terry, J.P. Hammond, and A.J. Thompson. 2012b. Association of gene expression data with dormancy and sprout suppression in onion bulbs using a newly developed onion microarray. Acta Hort. 969:169-174.

D'Angelo, C.J. and I.L. Goldman. 2018. Temporal aspects of vernalization and flowering in long day storage onion. J. Amer. Soc. Hort. Sci. 43:446-453.

El-Maarouf-Bouteau, H. and C. Bailly. 2008. Oxidative signaling in seed germination and dormancy. Plant Signal. Behav. 3:175-182.

FAO. 2016. FAOSTAT crops data. 4 Feb. 2018. $<$ http://www.fao.org/faostat/en/\#data/QC $>$.

Horvath, D.P., J.V. Anderson, W.S. Chao, and M.E. Foley. 2003. Knowing when to grow: Signals regulating bud dormancy. Trends Plant Sci. 8:534-540.
Lang, G.A., J.D. Early, G.C. Martin, and R.L. Darnell. 1987. Endo-, para-, and eco-dormancy: Physiological terminology and classification for dormancy research. HortScience 3:371-377.

Liu, X., Z. Deng, H. Cheng, X. He, and S. Song. 2011. Nitrite, sodium nitroprusside, potassium ferricyanide and hydrogen peroxide release dormancy of Amaranthus retroflexus seeds in a nitric oxide-dependent manner. Plant Growth Regulat. 64:155-161.

Mazzitelli, L., R.D. Hancock, S. Haupt, P.G. Walker, S.D.A. Pont, J. McNicol, L. Cardle, J. Morris, R. Viola, R. Brennan, P.E. Hedley, and M.A. Taylor. 2007. Co-ordinated gene expression during phases of dormancy release in raspberry (Rubus idaeus L.) buds. J. Expt. Bot. 58:1035-1045.

Mohamed, H.B., A.M. Vadel, J.M.C. Geuns, and H. Khemira. 2012. Effects of hydrogen cyanamide on antioxidant enzymes' activity, proline and polyamine contents during bud dormancy release in Superior Seedless grapevine buds. Acta Physiol. Plant. 34:429-437.

Née, G., Y. Xiang, and W.J. Soppe. 2017. The release of dormancy, a wake-up call for seeds to germinate. Curr. Opin. Plant Biol. 35:8-14.

Oracz, K., H.E. Bouteau, J.M. Farrant, K. Cooper, M. Belghazi, C. Job, D. Job, F. Corbineau, and C. Bailly. 2007. ROS production and protein oxidation as a novel mechanism for seed dormancy alleviation. Plant J. 50:452-465.

Pak, C., L.H.W. van der Plas, and A.D. de Boer. 1995. Importance of dormancy and sink strength in sprouting of onions (Allium cepa) during storage. Physiol. Plant. 94:277-283.

Pérez, F.J. and W. Lira. 2005. Possible role of catalase in post-dormancy bud break in grapevines. J. Plant Physiol. 162:301-308.

R Development Core Team. 2018. R: A language and environment for statistical computing. R Foundation for Statistical Computing. Vienna, Austria. 13 July 2019. <https://www.R-project.org> .

Shishido, Y. and T. Saito. 1977. Studies on the flower bud formation in onion plants III. Effects of physiological conditions on the low temperature induction of flower buds in bulbs. J. Jpn. Soc. Hort. Sci. 46:310-316.

Vergara, R. and F.J. Pérez. 2010. Similarities between natural and chemically induced budendodormancy release in grapevine Vitis vinifera L. Scientia Hort. 125:648-653. 